12

\title{
Наблюдение крупномасштабного дробового шума в сильноточном электронном пучке
}

\author{
(C) Э.Б. Абубакиров, А.Н. Денисенко, А.П. Конюшков, \\ Е.И. Солуянов, В.В. Ястребов
}

Институт прикладной фризики РАН, Нижний Новгород, Россия

E-mail: edward@appl.sci-nnov.ru

Поступило в Редакцию 11 мая 2018 г.

Экспериментально исследуется характер шумовой компоненты электронного пучка, формируемого взрывоэмиссионным катодом. Показано, что ее источником является крупномасштабный дробовой шум, связанный с проявлением эктонных структур в электронном пучке. Измеренные количественные характеристики эктонов находятся в хорошем согласии с ранее полученными данными и оценками.

DOI: $10.21883 /$ PJTF.2018.19.46676.17380

Согласно современным представлениям, взрывная эмиссия электронов, обеспечивающая формирование сильноточных пучков в подавляющем большинстве систем мощной электроники, имеет дискретный характер. В результате его проявления электронный поток представляет собой суперпозицию токовых импульсов („эктонов“), возникающих в случайный момент времени с характерной длительностью около $10^{-8} \mathrm{~s}$ и состоящих из порядка $10^{11}-10^{12}$ частиц [1]. Очевидно, что отличительной чертой такого процесса будет значимая шумовая составляющая электронного тока, имеющая характер дробового шума [2]. Если ток 
пучка представить в виде

$$
J(t)=\sum_{k} j_{k} v\left(t-t_{k}\right)
$$

(где $j_{k}-$ амплитуда одиночного импульса, возникающего в момент времени $t_{k} ; v(t)$ - функция, описывающая форму этого импульса), то, как известно (см., например, [3]), основные характеристики такого случайного сигнала могут быть вычислены как

$$
\begin{gathered}
\bar{J}=\alpha \bar{j}_{k} \int_{-\infty}^{\infty} v(t) d t, \\
D=\alpha \bar{j}_{k}^{2} \int_{-\infty}^{\infty} v^{2}(t) d t, \\
\rho(\tau)=\frac{\alpha \bar{j}_{k}^{2} \int_{-\infty}^{\infty} v(t) v(t+\tau) d t}{D} .
\end{gathered}
$$

Здесь $\bar{J}$ - средний ток пучка, $D-$ дисперсия тока, $\rho$ - нормированная корреляционная функция, $\alpha-$ средняя частота появления элементарных токовых импульсов.

Для простейших оценок примем, что все одиночные импульсы имеют прямоугольную форму с длительностью $\tau$ и несут одинаковый заряд $Q$. Тогда $j_{k}=Q / \tau$, средний ток пучка $\bar{J}=\alpha Q$, а среднее число элементарных импульсов, составляющих полный ток, $N=\bar{J} \tau / Q$ и дисперсия шума $D=\bar{J} Q / \tau=\bar{J}^{2} / N$ оказываются пропорциональными среднему току пучка. Корреляционная функция сигнала в рассматриваемом приближении, очевидно, имеет форму треугольника, ширина основания которого равна длительности элементарного токового импульса. Для указанных параметров единичного эктона при токе в несколько килоампер величина $N \sim 10^{3}$, и относительная амплитуда шумовой компоненты $\delta J=\sqrt{D} / \bar{J}=1 / \sqrt{N}$ составит единицы процентов. Представляется, что сигнал такого уровня может быть обнаружен экспериментально, что может стать дополнительным подтверждением существования обсуждаемых особенностей взрывной эмиссии электронов. Анализ токового сигнала в предположении его эргодичности может

Письма в ЖТФ, 2018, том 44, вып. 19 
быть проведен при помощи элементарных приемов статистической обработки - вычисления дисперсии и автокорреляционной функции для зависимости тока электронного пучка от времени. Задача настоящей работы состоит в измерении и анализе параметров шумов электронного пучка в условиях, характерных для устройств и систем сильноточной электроники.

Естественно, что при выборе экспериментальных условий проведения измерений шумовых характеристик взрывоэмиссионных электронных потоков ключевым моментом оказывается длительность токового импульса, которая должна существенно превышать длительность единичного эктона. При таких условиях наблюдаемый процесс может считаться квазистационарным, и для него возможно определение указанных выше характеристик. В связи с этим экспериментальные исследования были выполнены на базе сильноточного ускорителя стенда „Крот“ ИПФ РАН, позволявшего генерировать пучки релятивистских электронов с длительностью импульса до нескольких сотен наносекунд. Представляемая серия экспериментов была проведена при максимальном ускоряющем напряжении $800 \mathrm{kV}$ и максимальном токе пучка около $5 \mathrm{kA}$. Трубчатый электронный пучок диаметром $42 \mathrm{~mm}$ формировался в коаксиальном диоде с магнитной изоляцией с холодным катодом из нержавеющей стали. Электронный пучок транспортировался в однородном магнитном поле величиной около $2 \mathrm{~T}$ на расстояние $150 \mathrm{~mm}$, после чего осаждался на торцевой графитовый коллектор, соединенный с резистивным токовым шунтом. Ускоряющее напряжение измерялось емкостным датчиком, установленным перед вакуумным изолятором ускорителя. Осциллограммы тока и напряжения регистрировались цифровыми осциллографами (Tektronix TDS 3052) с максимальной полосой пропускания $1 \mathrm{GHz}$ и частотой выборок до $5 \cdot 10^{9}$ отсчетов в секунду. Для получения максимальной длительности импульса и минимизации уровня помех, связанных с работой высоковольтных коммутационных элементов, в описываемой серии экспериментов в ускорителе был реализован режим с затянутым передним фронтом ускоряющего напряжения $U$ и соответственно тока (рис. 1$)$.

Выделение шумовой компоненты тока для ее дальнейшего анализа проводилось следующим образом. Из цифрового сигнала, полученного осциллографом при максимальной частоте выборок $(5 \mathrm{GHz})$, вначале выделялся медленно меняющийся ток пучка $\bar{J}(\tau)$ (рис. 2,a) усреднением бегущим прямоугольным окном с шириной $40 \mathrm{~ns}$. Длительность

Письма в ЖТФ, 2018, том 44, вып. 19 


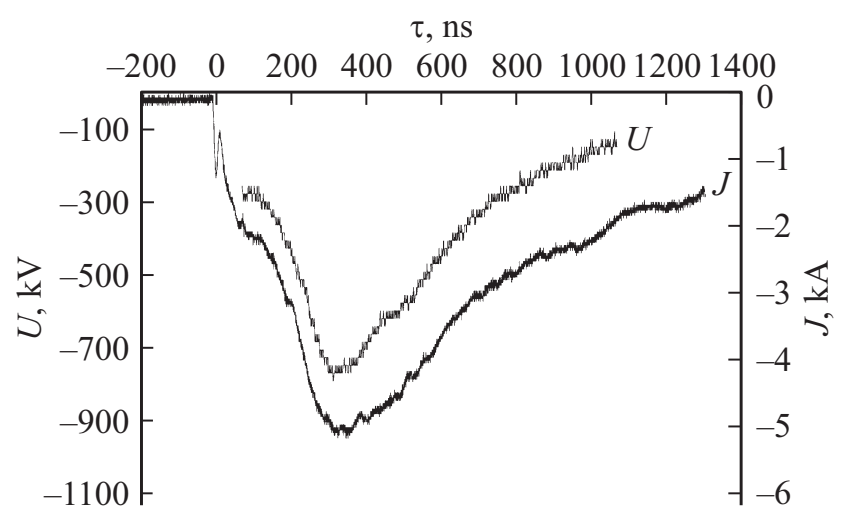

Рис. 1. Импульсы ускоряющего напряжения $U$ и тока пучка $J$.

окна для построения усредненного импульса тока выбиралась так, чтобы, с одной стороны, заведомо превысить характерный временной масштаб ожидаемых шумовых процессов $(10-20 \mathrm{~ns})$, а с другой учесть все медленные изменения в форме импульса электронного тока. Затем исходный сигнал центрировался вычитанием среднего тока. Существенно, что полученный шумовой сигнал наряду с собственными шумами электронного пучка содержит значительную примесь аппаратного цифрового шума, обусловленную не очень высокой разрядностью (9 bit) аналого-цифрового преобразователя осциллографа. В рассматриваемых условиях по существу реализована ситуация переоцифровки (oversampling, см., например, [4]), так как верхняя рабочая частота датчика тока вместе с сигнальным кабелем не превышает 150-200 MHz и соответственно намного меньше частоты следования временны́х отсчетов регистратора, поэтому снижение уровня цифрового шума может быть обеспечено за счет низкочастотной фильтрации полученных данных. В нашем случае такая фильтрация была проведена усредняющим окном шириной $3 \mathrm{~ns}$. Для полученного сигнала с нулевым средним значением $S(\tau)$ (рис. 2,a) затем вычислялись амплитудная дисперсия $D(\tau)(3)$ (рис. 2,b) и автокорреляционная функция (4) (рис. 3).

По результатам вычисления величины дисперсии шума в импульсе тока можно выделить три характерные области: 1) начальная часть (примерно до $300 \mathrm{~ns}) ; 2$ ) средняя часть ( 300-600 ns); 3) окончание 

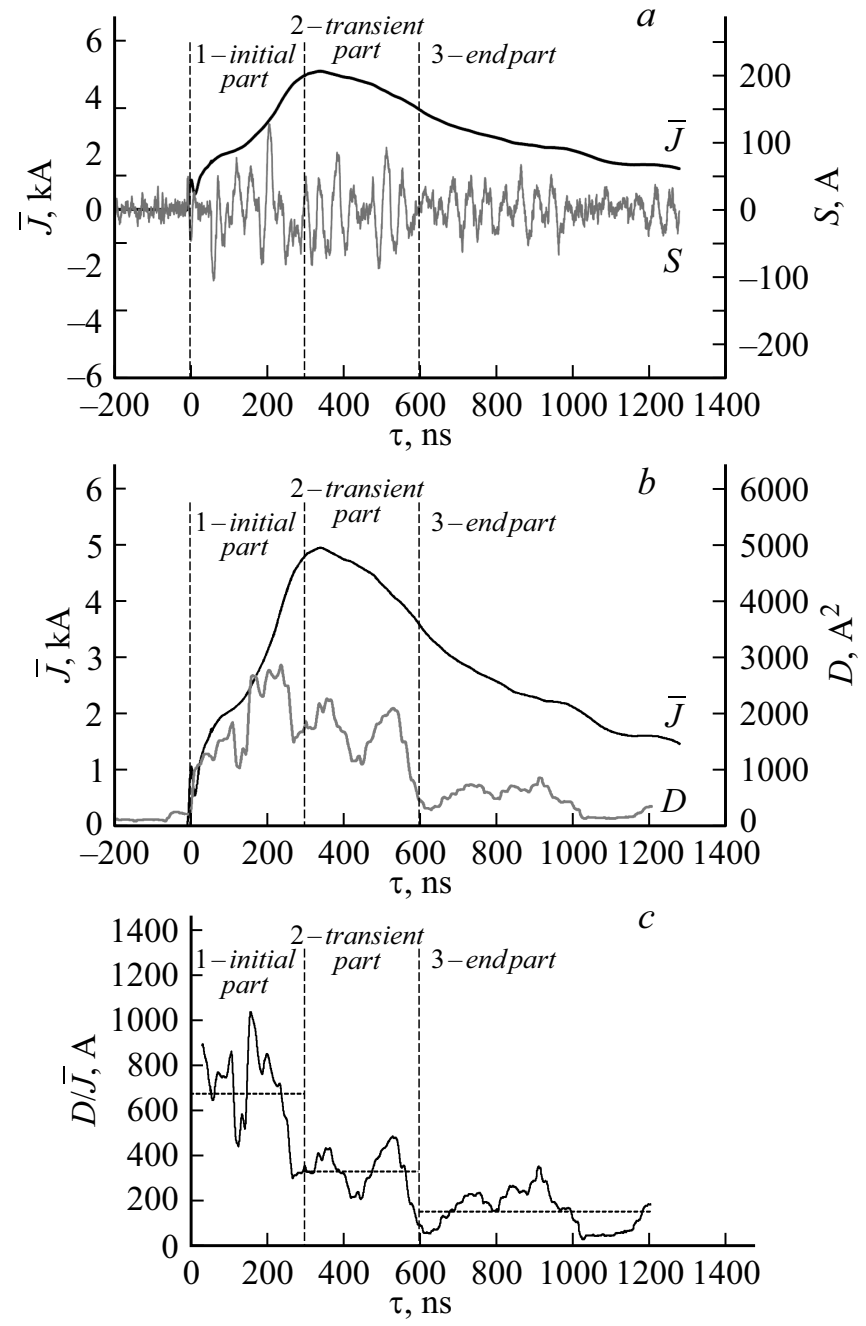

Pис. 2. $a-$ усредненный ток $\bar{J}$, шумовая компонента тока пучка $S ; b-$ дисперсия шума $D$ и усредненный ток $\bar{J} ; c-$ отношение дисперсии $D$ к усредненному току $\bar{J}$ (сплошная линия) и усредненные значения $D / \bar{J}$ для интервалов 1, 2, 3 (штриховые линии).

Письма в ЖТФ, 2018, том 44, вып. 19 


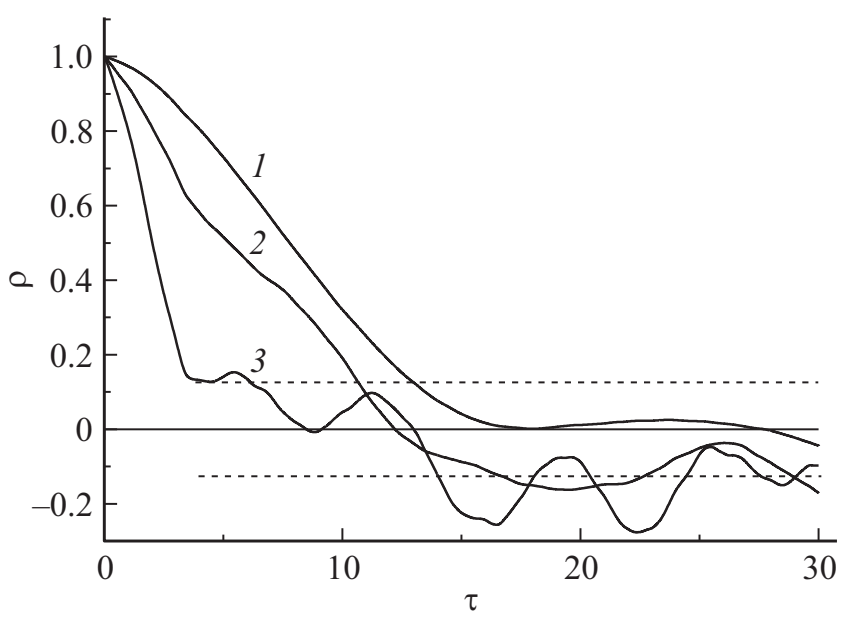

Рис. 3. Автокорреляционная функция шума на начальном (1), среднем (2) и конечном (3) участках импульса тока. Штриховыми линиями обозначены границы доверительного интервала: при нахождении рассчитанного значения автокорреляции вне этих границ можно с 95\% вероятностью утверждать, что ее истинное значение не равно нулю.

импульса тока (свыше $600 \mathrm{~ns}$ от начала импульса). В первой части отношение $D / \bar{J}$ наиболее близко к константе, на втором участке интенсивность шума падает примерно вдвое, на заключительном участке относительный уровень шумов оказывается еще ниже (рис. 2,c). По виду автокорреляционной функции можно определить, что на начальном участке импульса шумовой сигнал коррелирован с характерным временем $\tau \approx 17 \mathrm{~ns}$ (рис. 3, кривая 1 ). На последнем отрезке временна́я корреляция шума отсутствует (рис. 3, кривая 3), поскольку ширина автокорреляционной функции не превышает $3 \mathrm{~ns}$, т.е. величины, обусловленной предварительной низкочастотной фильтрацией исходного сигнала. На среднем участке (рис. 3, кривая 2) корреляционная функция шума имеет переходный характер по отношению к областям 1 и 3. Исходя из наблюдаемой картины можно предположить, что начальная часть импульса тока формируется взрывной эмиссией электронов с выраженным содержанием крупномасштабных порций электронов длительностью 15-20 ns. При больших временах процесса характер

Письма в ЖТФ, 2018, том 44, вып. 19 
тока меняется, что, скорее всего, связано с постепенным заполнением пространства между катодом и коллектором плазмой. Согласно [5,6], характерная скорость коллекторной плазмы может достигать $10^{8} \mathrm{~m} / \mathrm{s}$, и в использованной конфигурации за время порядка 200-300 ns наиболее быстрые фракции такой плазмы достигают катода. На этапе 3 через 500-600 ns после начала импульса формирование тока и его протекание по каналу, очевидно, полностью обусловлены катодной и коллекторной плазмой, и эктоны в его структуре не наблюдаются. Отметим, что для проверки физической обусловленности полученных результатов аналогичная процедура обработки была проведена и для сигнала с датчика ускоряющего напряжения $U(\tau)$ (рис. 1). Временна́я корреляция для шумовой компоненты этого сигнала обнаружена не была.

Рассчитаем параметры эктонов в условиях проведенного эксперимента исходя из характеристик электронного тока на участке 1 . Максимальная среднеквадратичная интенсивность шума здесь составляет $D=2.9 \cdot 10^{3} \mathrm{~A}^{2}$ при токе $\bar{J}=3.3 \mathrm{kA}$. Согласно приведенным ранее соотношениям, это соответствует одновременному существованию $N \sim 4 \cdot 10^{3}$ эктонов в пучке и при длительности элементарного токового импульса $\tau \approx 17 \mathrm{~ns}$ единичный эктон содержит около $9 \cdot 10^{10}$ частиц. Полученные значения хорошо согласуются с оценками, содержащимися в монографии [1], приведенными в начале настоящей работы.

Таким образом, результаты проведенных измерений шумовой составляющей тока электронного пучка могут быть интерпретированы как прямое проявление эктонных структур в потоках, формируемых взрывоэмиссионными инжекторами. Измеренные количественные характеристики эктонов (их число, объем и длительность) находятся в хорошем согласии с ранее полученными данными и оценками. Представляется, что рассмотренный подход, использующий вычисление основных статистических характеристик наблюдаемого крупномасштабного дробового шума, может быть использован для анализа динамики как взрывной эмиссии электронов в различных экспериментальных условиях, так и других физических процессов, в которых проявляются эктонные структуры, в том числе в электрических разрядах в вакууме и газе [7].

Работа выполнена при поддержке Российского фонда фундаментальных исследований (грант № 18-08-00926 А).

Письма в ЖТФ, 2018, том 44, вып. 19 


\section{Список литературы}

[1] Месяи Г.А. Эктоны. Екатеринбург: Наука, 1993. Ч. 1. 184 с.

[2] Абубакиров Э.Б., Конюшков А.П., Сергеев А.С. // Радиотехника и электроника. 2009. Т. 54. № 8. С. 1009-1014.

[3] Корн Г., Корн Т. Справочник по математике для научных работников и инженеров. М.: Наука, 1974. 832 с.

[4] Lyons R.G. Understanding digital signal processing. 3rd ed. Prentice Hall, 2010. $954 \mathrm{p}$.

[5] Зайщев Н.И., Кораблев Г.С., Шемякин Б.П. // Физика плазмы. 1981. Т. 7. № 3. С. $560-563$.

[6] Александров А.Ф., Галузо С.Ю., Зайцев Н.И. Кочелев В.И., Стрелков П.С., Федотов А.В., Шкварунеи, А.Г., Шмелев М.Ю., Энгелько В.И. // Релятивистская высокочастотная электроника. Сб. науч. тр. Горький: ИПФ АН СССР, 1988. B. 5. C. $163-182$.

[7] Месяи, Г.А., Баренгольи, С.А. // УФН. 2002. Т. 172. № 10. С. 1113-1130. 